\title{
Proofs, Reasoning and the Metamorphosis of Logic*
}

\author{
Jean-Baptiste Joinet \\ Université Panthéon-Sorbonne (Paris 1), Département de Philosophie. \\ C.N.R.S. (Paris 7), Équipe "Preuves-Programmes-Systèmes". \\ (joinet@univ-paris1.fr)
}

\begin{abstract}
With the "mathematical watershed", Logic had been transformed into a foundational theory for mathematics, a theory of truth and proofs - far away from its philosophical status of theory of the intellectual process of reasoning. With the recent substitution of the traditional proofs-as-discourses paradigm by the proofsas-programs one, Logic is now becomming a foundational theory for computing. One could interpret this new watershed as being "yet another technological drift", bringing Logic always closer to practical ingeneering, always further from the human intellectual process of reasoning. This article promote the dual point of view: enlightened by the contemporary analysis of the dynamic of proofs, which bring us to a new understanding of the semantic counterpart of processes operationality (including the links between semantic dereliction due to inconsistency and computational exuberance), Logic has never appeared so close to being, finally, the theory of reasoning.
\end{abstract}

Keywords: proof, computation, reasoning, philosophy of logic

\section{Introduction}

When it comes to giving a short definition of Logic, one frequently hears (sometimes even said by oneself) that Logic is "the theory of reasoning". Yet, all contemporary logicians know very well that Logic, as it is practiced today, is not properly about reasoning, but about proofs. Reasoning indeed is a human intellectual process, wheras proofs - as logicians, from Aristotle to Gentzen, have investigated them are discourses: oral or written, maybe only formalized, but discourses nonetheless.

This shortcut made by logicians when presenting their discipline (or perhaps more accurately said: "the fantasy" of logicians when they are representing to themselves the subject of their work) actually accompanied Logic, under various declensions, all along its history. Moreover, as starts in the XIXth century the process of gradual absorption of Logic

* Lecture given under title From Natural Deduction to the nature of reasoning, at the colloquium Natural Deduction organized by Luiz Carlos Pereira and dedicated to the work of Dag Prawitz, Rio de Janeiro, Brazil, July 2001. To appear in Luiz Carlos. Pereira, Edward Hermann Hauesler and Valeria de Paiva (eds.), Advances in Natural deduction (proceedings of the 2001 "Natural Deduction" conference, PUC, Rio de Janeiro, Brasil), Springer. 
by Mathematics, the view of Logic as being the "Theory of thought" itself continues to prevail ${ }^{1}$ and, even after the birth of proper Proof theory - actually by the pen of its promotor himself, David Hilbert -, it is reiterated: "The fundamental idea of my proof theory is non other than to describe the activity of our understanding, to make a protocol of the rules according to which our thinking actually proceeds" (Hilbert 1927, p. 475).

As those same logicians who present Logic choosing such words, in the meantime explicitely deny any psychological nature to logical principles, one sees that, underlying such formulations, stands the somewhat confuse idea that some kind of isomorphism or interface would hold between the rules "according to which our thinking proceeds" (or "has to proceed" as well ${ }^{2}$ ) and the rules linking sentences in our deductive discourses, so would hold between reasonings and proofs.

To clarify how reasoning and proofs however radically differ, it is useful to stress the three following specific points, more or less traditionnally emphasized when comparing the two notions. First, as it is a process, reasoning is of a dynamic nature, whereas proofs, as discourses and especially as texts, are static objects. Second, the intellectual, rational process of reasoning would presuppose the grasping of meaning, the mental representation of objects and structures, which is frequently summed up by speaking of the "semantic" or "contentual" aspects of reasoning, whereas proofs articulate sentences inferentially, according only to their shape, without regard for reference. Third, wheras a wrong proof is not a proof, incorrect reasoning still is reasoning.

\section{Dynamic character of reasoning versus Static character of proofs \\ Referential dimension of reasoning versus Inferential nature of proofs Indeterminacy of reasoning versus Correctness of proofs}

The reasons why contemporary Logic happens to deal with proofs instead of reasoning are numerous and varied. They are on the whole tied up with the fact that the silent human process of reasoning remains mainly observable through private mental self-introspection (or perhaps through some difficult to exploit but measurable side-effects, e.g., those produced by neural activity etc.), so that consciousness intervenes in its study, moreover both as an object and as a tool for observation. Even if, disregarding the usual problem of subjectivity's epistemological weakness, one acknowledged as relevant a question such as : "what information could introspection reveal to us concerning reasoning ?", the following specific difficulties - which match up with the three points stressed above - would have to be taken into account.

A first obstacle which prevents a direct approach to the intellectual process of reasoning is its introspective opaqueness. As David Hume

${ }^{1}$ G. Boole's Investigation of the Laws of Thought is here emblematic (Boole, 1930).

2 The fact that even the early intuitionistic tradition conceived explicitely Logic as a non psychological organon, as pure normativity (see for instance Kant, Logik, introduction, first section), does not obliterate its conception of Logic as the science of the laws of thought: that Logic studies "the rules according to which our thinking must proceed", rather than "how it proceeds" does not make any difference here. 
so nicely says, "it is remarkable concerning the operations of the mind that, though most intimately present to us, yet, whenever they become the object of reflexion, they seem involved in obscurity; nor can the eye readily find those lines and boundaries which discriminate and distinguish them" (Hume, 1748, ch. 1). The complex temporality and profound structure of reasoning, with its likely distributed interaction mechanisms, generally escapes our consciousness. Because we can perceive at best only some of the epiphenomenal, conscious effects of this internal process, we are unable to qualify directly its deep technological nature and have a direct apprehension of its dynamic. In strong contrast with this situation, rational discourses, and especially written ones, are by definition communicable and completely observable objects, and so are describable phenomena without any hidden parts, whose stability a contrario permit the manifestation and study of their structure.

Another obstacle is just simply that we are not clearly able to even recognize reasoning, unable "from the inside" to separate from the general stream of our thought some sub-process which would constitute its "rational part" and be independent of emotions and, especially when deliberation about practical choices is involved, of desires, fears and social determinations. In any event, when considering human thought in vivo, the traditional splitting of rational and non rational aspects seems rather arbitrary (this is also why the specific dynamic of wrong or mistaken reasoning is so unclear). In strong contrast with this situation, proofs as opposed to reasoning, or at least formalized proofs, are recognizable (for a text to be a proof is a decidable property). In particular, once a list of "logical rules" has been set down, the borderline between proofs and non proofs becomes unambiguously determined.

Finally, a relevant theory of reasoning should cover the referential dimension of the rational process, and hence would presuppose a theory of meaning as mental representation. This usually leads to unclear metaphorical formulations such as intimate "grasp" of semantic "content", private, direct mental contact with concepts, and to all manners of ambiguities that torment the philosophy of mind and consciousness. In strong contrast with this situation again, the logical analysis of discursivity opens onto a theory of objective, consciousness-independant meaning.

Among these issues, the last one constitutes the point of departure for analytic philosophy (in the true sense, i.e. restricted to epistemological topics), whose dismissal of psychologism in favour of semantic analysis parallels the methodological dismissal of reasoning as an object of study which de facto prevails in Logic. In a conference given in 1987, in which he extricated the roots of the analytic watershed in philosophy, Michael Dummett deepened the question of this methodological precedence of language over thought (Dummett, 1987). I am retaining from him, notably, the thesis that because there is something objective in meaning (as communicability shows) which is completely involved and kept within discursivity (as communication shows), the only objective knowledge about thought we can hope to grasp, if ever any, shall be proportionate to what is objective in discursivity: its structure, the norms which regulate our practice and use, or whatever ... 
In the present article, I will remain faithful to this analytic inspiration, which as I understand it, does not disqualify inquiries into reasoning, but merely postpone them as questions which could be posed only afterwards, as "secondary" questions. Instead of entering into the habitual debate over "truth conditions" which often comes down to searching outside of discursivity for an objective foundation for semantics (facts described by sets, worlds of concepts, human rational practices whose only criterion is to be the ones humans happen to learn, etc.), I would like to focus my investigations upon objective features of discursivity and proofs that have been revealed by recent prooftheoretical developments, beginning with Gentzen's seminal work and continuing through later work based on the so-called Curry-Howard correspondance between proofs and programs (i.e. the remark that the evaluation of simply typed lambda-calculus and the normalisation of minimal Natural Deduction are isomorphic), have revealed.

More specifically, my aim here is to evaluate to what extent these developments give new insights into the relationship between "reasoning" (the human rational mental process) and "proofs" (structured inferential texts). I will tackle this question by successively taking up the three particular points recalled above which differentiate reasoning and proofs, examining in each case how to articulate them in light of recent proof-theoretical investigations (in particular those from the so-called proofs-as-programs paradigm).

\section{Dynamic character of reasoning versus Static character of proofs}

Whereas reasoning is a process, and thus inseparable from its dynamic, proofs, as usually taken into account by formal Logic, are traditionally apprehended as texts, and at first glance lack any true dynamic dimension. Indeed, one occasionally finds the absence of dynamic used against the formalist perspective in Logic, which is then typically blamed for being only occupied with cold, lifeless, static things, thus incomparable to human reasoning ${ }^{3}$. Is it so true, however, that contemporary Logic when working on proofs, does not deal with the dynamic?

First, there is still the old idea (dating back to at least the Aristotelian view on syllogisms) that some dynamic is involved when one is accomplishing a deductive step or reading a structured inferential discourse, insofar as one is indeed moving from sentence to sentence following the orientation of the deduction. Nevertheless, even those who consider inferential steps as imitating to a certain extent steps from idea to idea (echoing the famous Cartesian description of reasoning in the Regulae, in which the mind travels through an ordered chain of ideas, from distinct representation to distinct representation), do not assimilate the dynamic involved to the general, complex process by which an individual deliberates, deduces, and modifies his or her abstract representations and concrete judgments.

\footnotetext{
3 See Descartes, Hegel, Brouwer: actually, it is the anti-formalist topos par excellence.
} 
There is, however, a second axis through which contemporary Logic investigates directly what is undoubtedly a part of the dynamic process of reasoning, namely heuristics. In a general sense, heuristics covers the search for proofs of a given sentence from a given theory, hence includes the process of determinating which relevant sentences should be proven beforehand in the search for success. Modern formal Logic developed numerous techniques for tackling the heuristics challenge, importing dynamic features into proof theory. To what extent can we conclude that a dimension of human reasoning is here modeled? Of course, the way the heuristic dynamic is realized at the technical level (often using specific ad hoc formal systems suited to heuristic strategies, instead of systems such as Natural Deduction whose devising from the start was explicitly guided by the aim to imitate, in some respects, the way mathematicians "naturally" happen to write their proofs) renders difficult any comparison of the human heuristic with an artificial one. Moreover, one could object that contrary to the natural human heuristic dynamic which is part of the rational process, the artificial one stays outside of the world of proofs, since with respect to the search process, the produced proofs simply appear as outputs a priori not involving any tracks of the process by which they have been produced, so as by-products external to the dynamic.

Another kind of dynamic, though, also occupies the logical stage, namely proofs' normalisation, that process (first studied by G. Gentzen) by which proofs are converted into analytic ones (but more generally other processes of proofs' conversion such as, for instance, elimination, in relevant cases, of non constructive components of proofs), and which implements a dynamic completely internal to the proofs' world. Besides the well-known epistemological value of the convertibility of proofs (which legitimates abstract and - when applicable - non constructive detours as safe proof-theoretic means), the rational flavour of the conversion process itself deserves to be pointed out.

First of all, one has to observe that proofs, as subject to conversion processes, acquire a radically new status. In this light, the basic components of proofs, logical rules, become, literally, programming instructions: they determine the precise way evaluation works and control the operational destiny of the conversion process. That proofs have been historically first modeled, from a static point of view, as texts, turns out to be but an epiphenomenal feature, beyond which appears their true nature as operators acting on rational resources. (Incidentally, one may remark that in fact, even as static objects - though now enlightened by the dynamic perspective and adapted to it -, proofs do no longer really fit within the proofs-as-discourses view. Indeed, in recent proof systems like J-Y Girard's proof-nets (Girard, 1987, 1995, 1996), their correctness depends on global geometrical properties of the proof-structures, and so is not a locally checkable condition. Such an account of proofs is very far from what logicians have been accustomed to, namely textual structures linking rules of inference picked out from a catalogue determined in advance, and of which Hilbert's style systems or sequents' derivation systems are paradigmatic instances).

Moreover, like ideas, proofs also happen to live very well together. Their "sociability" is effective not only at the static level through 
their modularity (proofs can be associated, combined, reused), but also dynamically, as normalisation precisely shows by ensuring, in case their combination increases the level of abtraction, that analytical avatars of the abstract proof still remain "kept in sight".

Thus far from being absent from proof-theory, the dynamic today has become the central object of mainstream logical investigations, and a conceptual unification of both dynamics - that of heuristics and that of proofs' conversion - is even at hand ${ }^{4}$. For all that, is the newfound centrality of the dynamic enough to conclude that bridges between theory of proof and theory of reasoning are now in sight ? A crucial indication could come from assessing to what extent the intrusion of the dynamic sheds light on the semantic aspects that reasoning reputedly involves.

\section{Referential aspects of reasoning versus inferential nature of proofs}

The uses of sentences fall into two apparently discrete modes. In the inferential mode, which is completely internal to discourse, sentences are used and linked together according only to their form, no matter the interpretations they otherwise could receive. In the referential mode, discourses are "discourses about" - they refer to -, and sentences then are used according to interpretations of their components by referents external to discourse. The use of sentences in proofs $^{5}$ provides the paradigmatic instance of the inferential mode. At the opposite extreme, because reasoning involves the mental handling of representations (of individuals, structures, concepts, or wathever), it usually is taken to be an instance of the referential mode. This traditionally accepted dichotomy, whose unifying principle is given by 'completeness theorems', actually has been deeply disturbed by (relatively) recent proof-theoretical advances (which happen to confirm in a striking way the relevance of Michael Dummett's remarks about the replacement of the notion of truth by the notion of proof as the central notion of the theory of meaning (Dummett, 1978)).

As for the prevalence of the inferential mode in proofs, one first has to notice that proofs, at least analytic (cut-free) ones, being, so to speak, extensional descriptions of objects or structures, clearly involve 'internal' denotation in the sense that they are but explicit constructions. One may first illustrate this by considering the particular shape of the analytic proofs of the type of natural integers (or other data types) in second-order intuitionistic Natural Deduction, namely "Church's integers". In this first example, the reference is so to speak inferentially built: such a proof is but the standard construction of a given integer (in the Leibniz-Peano sense). Another example of such an inferential explicitation of reference is given by the fact that whenever they are

\footnotetext{
4 See J-Y Girard's Ludics (Girard, 2001).

5 i.e. the various ways sentences are concretely handled in proofs (for instance, in Natural Deduction systems, by such or such "introduction" and "elimination" rules).
} 
in a relevant form, analytic proofs literally describe models: think of the model (the counter-model) that one literally 'reads' in the analytic (pseudo-)proof of an (unprovable) sentence generated by the (here failed) proof-search strategy used to prove the completeness theorem for first-order $\operatorname{Logic}^{6}$.

But, of course, the main point is elsewhere. Beyond this first kind of 'inferential reference', subtler accounts of proofs denotation have been elaborated by contemporary proof theory, gradually realizing, at various levels and with always more relevance, Heyting's beautiful original idea of considering proofs as functions and not simply as texts.

All these semantics are semantics of proofs (as opposed to semantics of sentences), which means that they approximate the nature of proofs as operators, catching their "being" as a "doing" 7 and revealing the nature of argumentative dealings as computations over rational resources (one recovers a universal operational meaning for sentences themselves, in case one is able to identify an operational behavior common to all proofs of a given sentence ${ }^{8}$ ).

At one pole, there is denotational semantics, where proofs are directly interpreted by functions belonging to suited set-theoretically defined functions spaces (in such a way that the interpretation of proofs is invariable during their normalisation). However, semantics of this kind keep only a shadow of proofs' dynamic: interpretation remains too extensional and equalizes computations that should not be considered as equal and so, semantics fail to spot many intentional dynamic features.

At the opposite pole, one finds operational semantics, the "behaviourist" approach of the dynamic of proofs. A function is simply identified with the paraphrastic description of the set of all computations steps (of all the sequences of states describing possible evaluations). The defect in this case is converse to the former one: too much intentionality also kills off intentionality.

Between these two extreme poles, there is the more recent interaction semantics ("game semantics" and "ludics"), where dynamic (evaluation) is interpreted as a structured interaction, described, in the language of Game-Theory, as plays between processes whose rules structure their inter-communication (and where "conversion dynamic" and "proof-search dynamic" tend to coincide).

In the other hand, as regards the prevalence of the referential mode concerning reasoning and its "semantic contents", etc., one must conversely observe that, while one does not assume something like a "mental discourse" that would be used when we are reasoning, the words "referential mode", which, as they have been introduced above, do only apply to discourses (not to "psychological entities" like ideas), happen to be used here improperly and mistakenly. Yet, to assume the existence of such a mental discourse somehow amounts to reduplicating the

\footnotetext{
${ }^{6}$ See for instance: Wainer and Wallen (1992).

7 "Church's integers" for instance, to continue with our example, dynamically appear as iterators.

8 Particular case of Krivine's "specification problem" (Danos and Krivine, 2000).
} 
speaker "inside" the speaker, thus adopting the old vain prosopopeia of intellectual faculties (whose aporetic nature, being rather commonly acknowledged, do not require here deeper presentation ${ }^{9}$ ). Only one way remains thus open: as soon as reasoning is concerned, we must completely reverse the traditional view about meaning, now looking at it as an issue addressed, no longer to sentences, but to processes themselves.

Of course, at first sight, such a radical shift seems to present more new problems than answers. First of all because as we have seen, the notion of "process" seems very tricky compared to that of "discourse" (at least insofar as reasoning is concerned), but also because the account of meaning one henceforth must give in order to escape the above "reduplication" difficulty could no longer be in terms of something external (as referents did with respect to discourses), but of something completely internal to the world of processes. Thus how could meaning generation be a property of processes? As vague as the notion of process may be, it certainly is in their nature not only to evolve (amongst the effects produced by a process appear its own transformations), but also to produce effects on other processes, mutual effects. In short, processes act and interact. Whatever its technological matter, whatever its implementation, the essence of a process is completely involved in its (potential) dynamic behavior: not only its own possible destinies under evaluation, but also the full set of possible operational effects it will occasion in all possible processes' interaction contexts. With respect to semantics, the answer brought by processes is thus of a radically new kind, which implements the performative way of meaning ${ }^{10}$ : doing, is the way processes speak.

Enlightened by the theory of proofs' dynamic, the inferential mode is thus in the end far from being non referential. Moreover, the kind of semantics onto which it opens, do not incur the two major reproaches that the usual modelings of reference do, namely to award no proper status to abstraction (now caught by computational complexity and non analyticity of proofs) and to offer an evaluation-independant approach to sense. In that connection, it is not the least of the virtues of the operational viewpoint in Logic that it sheds light not only on the analogy between proofs conversion and rational dynamic, not only on the inseparability of evaluation and sense, but even finally, as we are going to see now, on nonsense itself.

\footnotetext{
9 For a synthetic presentation of the sophism of "homoncules", see Pinkas 1995. About the "mental language" thema, see the beautiful book of Claude Panaccio: "Le discours intérieur" (Panaccio 1999).

10 I am borrowing from Danos (1990), himself inspired by Austin terminology, the idea of "performativity" , there used to qualify formal systems of proofs (like Krivine's $A_{2}$ ) where proofs indeed do (as programs) what the sentences they are proving "say" they should. For a more complete presentation and a defense of the concept of "performativity" applied to the semantics of proofs, see J.-B. Joinet, "Sur le temps logique", to appear in Logique et Interaction: Géométrie de la Cognition, J.-B. Joinet (ed.), Publications de la Sorbonne, 2006 (or 2007), Paris.
} 


\section{Correctness of proofs versus Indeterminacy of reasoning}

Reasoning in its general sense includes "wrong reasoning": mistakes, inconsistencies, errances, partiality, revision, interruption of reflection (because of abstruseness, loops to be broken, breaks to be taken, emergencies or whatever). By contrast, when proof theory was born at the beginning of last century, it was certainly not conceived of as including a theory of "non-proofs". Admittedly, the definition of formal proof systems that was provided by Logic supplied decision procedures separating texts accepted as proofs from other texts (provided the catalogue of rules is set). Although such a definition thus gave a de facto account of what "wrong proofs" are, their status remained that of garbage, not worth studying.

On these issues, the recent transformations in proof theory, now focused not so much on proofs themselves as on their dynamic, have broadened the outlook. The first though not the main point: because once the dynamic is concretely implemented, occasions for new kinds of mistakes may happen (in the real world, "un pas suffit pour un fauxpas"11; the evaluation could be interrupted etc). But overall, because of the status of proofs having changed, that of non-proofs, as a result, also has changed. From the proofs-as-programs viewpoint, a proof is no longer just a normed discourse, but a process whose operationality, tamed by Logic, is "under control". This becomes clear once one tackles proofs in computational terms: then a proof is a typed program, whose typability ensures that certain computational properties are satisfied (typically: termination, complexity bounds ${ }^{12}$, etc). Nevertheless untypable programs - those processes that do not correspond to proofs and that Logic fails to civilize - share with proofs their life as dynamic entities, their computational nature.

The new status that paradoxes acquire when scrutinized from the belvedere of the dynamic illustrates this radical change of viewpoint. The traditional reasons given to reject inconsistency, from Aristotle (dialogue become impossible) to modern Logic (every sentence becomes provable: all of them becoming equivalent, there could be no meaning), may now be unifyingly reformulated: inconsistency permits the typing of infinite - and even infinitely silent - computations ${ }^{13}$. The point with paradoxical theories is thus not so much that they violently equalize sentences, but that they produce computational exuberance, and leads to the degeneration of the evaluation of meaning.

\section{Conclusion}

The "naturalness" of Natural Deduction, akin to the way mathematicians historically have come to write their proofs, is the fruit of the imitative intention which governed its settlement by Gerhard Gentzen. Any extension of such a "figurative" attempt to reasoning itself stumbles over our inability to describe and even recognize what

\footnotetext{
${ }^{11}$ Carlo Goldoni (original italian version not recovered).

12 See Girard, 1998 and Danos and Joinet, 2002.

13 Adding $X=\neg X$ ( $X$ an atomic formula), non-normalisable $\lambda$-terms like $\Delta \Delta$ become typable.
} 
we are suppose to imitate. Because no criterion for the success of the imitation enterprise is offered when the reasoning is on the line of sight, simulation, imitation, figurativity have properly no methodological meaning. The best one can try then is, on the one hand, to recover at most some of the features of this globally uncatchable phenomenon (e.g. dynamic features), and on the other hand, to reach some more abstract form of naturalness appearing in "aesthetic" properties like simplicity, universality, non-diffuseness (cf. proof nets' sobriety), harmony (input / output rules (Prawitz, 1977)) or in "architectonic" properties like modularity, transparent cutting-out into subsystems, representational strength (complexity)...

Of course the need for such an "artificial naturalness", could be interpreted as the sign that, ever further from human thought, the drift of Logic - from its philosophical origins to mathematics, and now from mathematics to theoretical computer science - henceforth is, and with no return, consummated. Logic would just have become a foundational theory of computing, a technical device for designing and studying computation and programming languages ${ }^{14}$. In this connection, the current extension of the Curry-Howard style approach to new programming devices oriented toward communication (Danos and Krivine, 2000) instead of usual recursive computation of data (so ever closer to technological issues, ever further from what was, century after century, the central concern of Logic: rational thought) seems somehow to confirm this metamorphosis of Logic toward technology.

However, the Logical Foundations of Computing could just as well be dually seen "from the other side of the isomorphism" as Computational Foundations for Logic ${ }^{15}$. From this complementary viewpoint, where studied objects are dynamic ones, with an operationally, interactively built-in evaluation semantics, and among which even paradoxes receive a relevant dynamic status, Logic has probably never appeared so close to being the theory of reasoning.

\section{Acknowledgements}

I would like to thank Gilles Dowek (french National Institute for Research in Computer Science and Automatics, I.N.R.I.A.) for the friendly and serious moments we spent, walking and talking in the tropical garden of the PUC university campus in Rio, John Tain for his help concerning english and François Rivenc for his remarks and advices about an earlier version of this article. My participation to the conference Natural Deduction, Rio 2002 was supported by the french National Center for Scientific Research (Proofs-Programs-Systems team) and the brazilian Ministery for Research.

\footnotetext{
14 Indeed, it has for instance given birth to programming languages like Prolog, Caml, etc.

15 At first restricted to propositional minimal Logic, the Curry-Howard style approach has been extended now to almost all parts of Logic, notably to first and higher order classical Logic and ZF set theory (Girard, 1991; Parigot, 1992; Krivine, 1994; Danos, Joinet, Schellinx, 1997; Krivine, 2001).
} 


\section{References}

Boole, G., Investigation of the Laws of Thought (1930)

Danos, V., La logique linéaire appliquée à l'étude de divers processus de normalisation (principalement du lambda-calcul), PhD thesis in Mathematical Logic, (Proof theory), Université Paris 7, Paris (1990)

Danos, V. and J-B. Joinet, Linear Logic and Elementary time, Information and Computation (2002).

Danos, V., Joinet, J-B. and H. Schellinx, A new deconstructive logic: linear logic, Journal of Symbolic Logic, 62 (3), pp. 755-807 (1997)

Danos, V. and J-L. Krivine, Disjunctive tautologies and synchronization schemes, Proceedings of CSL'2000, LNCS 1862 : 292-301 (2000)

Dummett, M., The philosophical basis of intuitionistic logic, Truth and other enigmas, Duckworth (1978)

Dummett, M., Origins of analytic philosophy (conference given in Torino in 1987, first published in German). Edition consulted: Les origines de la philosophie analytique, Gallimard, Paris (1991)

Gentzen, G. Untersuchungen über das logische Schliessen, Mathematische Zeitschrift, 39, 176-210, 405-431 (1934)

Girard, J-Y., A new constructive logic: classical logic, Mathematical Structures in Computer Science, 1(3), 255-296 (1991)

Girard, J-Y., Linear Logic, its syntax and semantics, Advances in Linear Logic, JY.Girard, Y.Lafont, L.Regnier eds., London Mathematical Society Lecture Notes Series 222: Cambridge University Press (1995)

Girard, J-Y., Proof-nets: the parallel syntax for proof-theory, Logic and Algebra, Ursini and Agliano eds.: Marcel Dekker, New York (1996)

Girard, J-Y., Light Linear Logic, Information and Computation 143 (1998)

Girard, J-Y., Locus Solum, MSCS (2001)

Hilbert, D., The foundations of mathematics (1927), From Frege to Gödel, a Source Book in Mathematical Logic, 1971-1931, ed. by Jean van Heijenoort, Harvard University Presss (1967)

Hume, D., An enquiry concerning human understanding (1748)

Joinet, J-B., Sur le temps logique, Logique et Interaction: une Géométrie de la Cognition (J.-B. Joinet ed.) Publications de la Sorbonne, to appear in 2006 (or 2007

Krivine, J-L., Classical logic, storage operators and second order lambda-calculus, Annals of Pure and Applied Logic, 68, p. 63-78. (1994)

Krivine, J-L., Typed lambda-calculus in classical Zermelo-Fraenkel set theory, Archive for Mathematical Logic, 40, 3, 189-205 (2001)

Panaccio, C., Le discours intérieur: de Platon à Guillaume dOckham Editions du Seuil, Paris, 1999

Parigot, M., Lambda-mu calculus: an algorithmic interpretation of classical natural deduction, Logic Programming and Automated Reasoning, A. Voronkov ed., LNAI 624, Springer Verlag, p.190-201 (1992)

Pinkas, D., La matérialité de l'esprit. Un examen critique des théories contemporaines de l'esprit, La découverte, Paris (1995)

Prawitz, D., Meaning and proofs: on the conflict between classical and intuitionistic logic, Theoria, Krister Segerberg ed., Vol. XLIII, Part 1, 1-40., Stockholm (1977)

Wainer S.S. and L.A. Wallen, Basic proof theory, S.S.Wainer, P.Aczel and H.Simmons eds, Proof theory: a selection of papers from the Leeds proof theory programme 1990, Cambridge University Press (1992) 
Address for Offprints:

J-B Joinet

UFR de Philosophie

Université Paris 1 (Panthéon-Sorbonne)

17 , rue de la Sorbonne

75231 Paris cedex 5

France.

\section{Author's Vitae}

$J$-B. Joinet

Specialist of Proof theory and Logical foundations of computer science, the author is associated professor in Logic and Philosophy of Logic at University PanthéonSorbonne (Paris 1), where he is responsible for the Licence de Logique (teaching program in Logic). After having been member of the Mathematical Logic team (CNRS, University Paris 7), he integrated the Proofs-Programs-Systems team (PPS, CNRS, University Paris 7), a laboratory where logicians and computer scientists co-operate. For the philosophical side, he is co-ordinator of Logique et Interaction: vers une Géométrie de la Cognition, a scientists and philosophers research group about the computational foundations of Logic and their extension to other scientific fields (Physics, Biology, Economics). He is associated member of Philosophies contemporaines (Expérience et Connaissance, EXeCO), a research team of University Panthéon-Sorbonne. He is member of the Groupe Histoire, Philosophie, Sciences de l'École Normale Supérieure de Paris. 